\title{
REFLEXIONES SOBRE EL ENVEJECIMIENTO Y LA CALIDAD DE VIDA
}

\author{
Ors Montenegro, A; Laguna Pérez, A. \\ Departamento de Enfermería Universidad de Alicante • Apdo 99/E-03080 Alicante.
}

\section{RESUMEN}

El envejecimiento es individual, es el resultado de la combinación de nuestra carga genética y la forma de adaptarnos al teatro de nuestra vida. La adaptación satisfactoria, la alta autoestima, la libertad, la felicidad, el bienestar guardan una relación con la buena calidad de vida en general. Los factores que consideramos mas im-portantes para una buena calidad de vida en la vejez serían: el grado de funcionalidad física, psíquica y social, la muerte de un miembro de la pareja y el estado de salud percibida

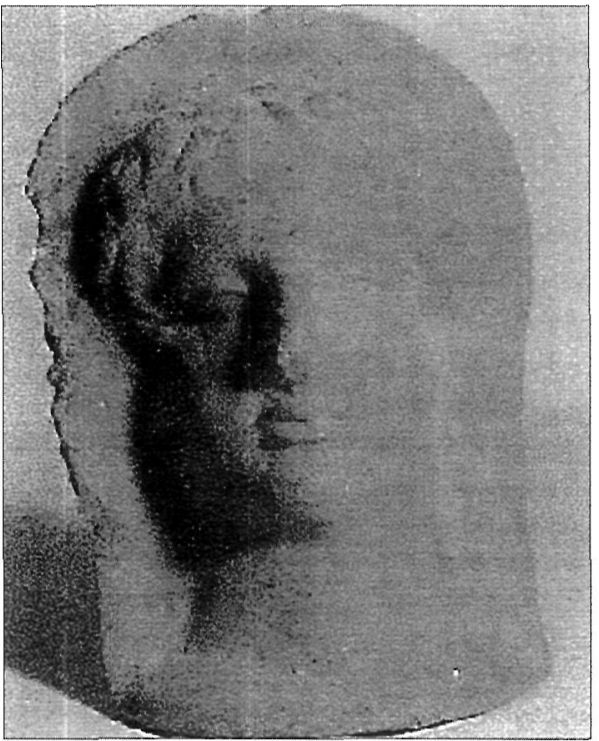

Cabeza votiva etrusca III-II A.C.

Palabras clave: teoría de los cuidados, enfermería geriátrica, calidad de vida.

\section{CONSIDERATIONS ABOUT AGING AND QUALITY OF LIVE}

\begin{abstract}
The way one ages is always personal. It is the result of one's own genetic load together with his/her adaptation to the life stage.

A satisfactory adaptation, self-respect, freedom, happiness and wellbeing, bear a strong relationship to the quality of life in general terms. Factors we consider essential for good quality of life in old age (elderly) are: physical, psychical and social fitness; a long and happy partnership with a loved-one, and self-perception of the state of one's health.

A good financial situation in old age, could sometimes be less significant, if compared with their former times in life when they have lived with more limited resources. It is not so important either when there is a social support such as NHS and/or certain bonuses which guaranty satisfaction for their basic needs such as food, clothing and housing for life.
\end{abstract}

\section{INTRODUCCIÓN}

El envejecimiento es un proceso de cambio biopsicosocial que se presenta mucho antes de que sus manifestaciones den aspecto de senecto o viejo. El envejecimiento es un hecho universal, individual, constante, irregu-lar, irreversible asincrónico y deletJreo.

El hombre se ubica en un ambiente, intercambiando influencias con él, de tal suerte que el envejecimiento viene a ser una forma de adaptación para sobrevivir. (Langarica, 1985)

El nivel de una hormona en los líquidos corporales y las consecuencias de su estímulo representan la suma de diversos procesos fisiológicos. Cada uno de ellos puede modificarse dentro de ciertos límites determinados por mecanismos genéticos intrínsecos y perturbaciones ambientales. El proceso de envejecimiento depende de factores intrínsecos al individuo o endógenos y factores ambientales o exógenos a él. Lo que afecta no es la conducta cotidiana del organismo con su medio, sino sus facultades, sus posibilidades de enfrentarse a una situación insólita tanto de origen biológico, psicológico o social.

Los cambios biopsicosociales no son sinónimo de enfermedad. A veces resulta difícil establecer los límites entre cambios estructurales y funcionales y enfermedad. De la misma manera que es difícil diferenciar entre salud y enfermedad. En muchos exámenes de salud se han encontrado alteraciones que no habían sido percibidas por las personas que las padecían. «El sano es un enfermo 
que ignora su condición», bien porque no sepa de ella o porque se haya adaptado y le permita vivir de forma libre, independiente y satisfactoria. La persona mayor sana, viejo joven o persona senecta sana es aquella que el paso de los años, más de 65 cumplidos, no le afecta para vivir de forma libre, independiente y gratificante. La persona mayor enferma, persona geriátrica, mayor o senecta enferma pierde la libertad para vivir como quiere pues la enfermedad tiende a la cronicidad y a la dependencia .

La salud significa vida y la vida nunca está terminada, completa siempre es mejorable. El término salud significa una escalada constante de mejoras en todos los niveles, una reivindicación diaria, una búsqueda de bienestar.

Desde la perspectiva ecológica la salud y la enfermedad dependen de numerosas variables. La salud vendría dada por la adaptación satisfactoria del hombre con su medio.

El hombre como ser vivo no tiene otra alternativa «envejecer y/o morir», para envejecer necesita asumir los cambios, las modificaciones e incluso los deterioros y buscar estrategias para «vivir» satisfactoriamente. Uno de los parámetros que mide el estado de salud en la última etapa evolutiva del hombre es la «funcionalidad». La funcionalidad física es la que más valora la persona mayor y las que quiere mantener mientras viva, de la psíquica, desea «un instante de lucidez más que de vida» $y$, de la funcionalidad social precisa suficiencia económica y relaciones sociofamiliares y en su defecto políticas adecuadas de pensiones, actividades culturales y ambientales que le faciliten su adaptación al ambiente y le garanticen un bienestar míRnimo «necesidades básicas garantizadas» (alimentación, vivienda, vestido, salud, cultura y ocio). El apoyo social interesa en relación a la salud y longevidad, las personas necesitan amar y sentirse amadas. La necesidad de sentirse amados parece no decrecer con los años, si acaso, aumentar. El estado de salud sí disminuye cuando la persona está desprovista de amor, de relación entre pareja, entre hijos y/o amigos. Lo confirma un estudio longitudinal de Oleen et al, 1991 llevado a cabo en Dinamarca con ancianos, el 5\% tenía más de 80 años y aparecía una relación entre las enfermedades cardiovasculares y mortalidad con el número de contactos anuales con hijos, hermanos y amigos. La integración social retrasaba la mortalidad en general y la cardiovascular en particular. Whestone y John 1991, encontraron que la carencia de apoyo familiar o conyugal disminuía la motivación del anciano para cuidarse. La calidad de vida, ha sido estudiada desde diferentes disciplinas. Socialmente calidad de vida tiene que ver con una capacidad adquisitiva que permita vivir con las necesidades básicas cubiertas además de disfrutar de una buena salud física-psíquica y de una relación social satisfactoria.

Entre los investigadores no hay consenso en la definición de «calidad de vida». Un concepto que involucra muchas variables subjetivas satisfacción, felicidad, autoestima ... es difícil de medir. Las variables objetivas son de medición más fácil la economía, el nivel sociocultural, los déficits funcionales, problemas de salud... Las definiciones que se han dado a este concepto son más un refle- jo de orientación profesional y áreas de interés de los distintos autores que análisis rigurosos del concepto de calidad de vida (Richart 1993)

Los investigadores con orientación clínica suelen definir calidad de vida en términos de salud y/o de discapacidad funcional. Por ej. Kaplan, define calidad de vida como el impacto de la enfermedad y la incapacidad sobre el funcionamiento diario. Ferruci diferencia la incapacidad funcional de la enfermedad. Dice que para la enfermedad deben emplearse estrategias terapéuticas y para la incapacidad terapias de rehabilitación y de apoyo social. Ribera,1992 nos aporta que no hay duda que la variable salud es la de mayor peso en la percepción de bienestar de los ancianos y, que los déficits de salud constituyen el primer problema para ellos.

- Según Andrews y Witney, «La calidad de vida no es el reflejo de las condiciones de los escenarios físicos, interpersonales y sociales, sino cómo dichos escenarios son evaluados y juzgados por el individuo».

- Según Kaplan, es el impacto de la enfermedad y la incapacidad sobre el funcionamiento diario.

- Según George y Bearon, satisfacción por la vida es autoestima, nivel general de salud y capacidad funcional.

- Según Bulpitt y Fletcher, sería la libertad, felicidad, seguridad económica deseos de realizarse, actividad y bienestar.

- Según Pearlman y Uhlmann, funcionamiento físico, social, emocional, satisfacción vital y bienestar.

- Entendemos como calidad de vida la satisfacción de vivir con libertad y bienestar, es decir con un funcionamiento físico, social, económico, emocional, que nos permita lograr todos nuestros deseos o nos resigne encontrándonos satisfechos, en paz, queridos, consolados.

Creemos que los factores que influyen en la calidad de vida, de las personas mayores, serían los siguientes:

- La independencia funcional: el grado de funcionalidad física, psíquica, económica. La persona independiente tiene mayor calidad de vida, que aquella que depende de su familia y/o de la sociedad.

- La vida en su casa, con los suyos, con su pareja. La pérdida de un miembro de la pareja tiene una repercusión negativa en la calidad de vida.

- La relación familiar, social. La soledad objetiva o subjetiva es un factor negativo en la calidad de vida.

Hemos revisado la bibliografía referenciada al final del trabajo y realizado unas entrevistas abiertas a profesionales de la enfermería y la psicología que trabajan en el campo de la calidad de vida y la vejez y, que explicamos a continuación para apoyar nuestras creencias

PRIMERA ENTREVISTA. Para el primer entrevistado «La calidad de vida» es un concepto multidimensional que cubre aspectos subjetivos y objetivos y, que tiene que ver con mucho con la satisfacción vital, la salud y la capacidad funcional, sobre todo con la «salud percibida». 
Los dos factores importantes son la salud percibida y la capacidad funcional entendida como autonomía.

La salud percibida se puede medir con preguntas, cuestionarios y la capacidad funcional con escalas específicas validadas para ello, por ej. el Perfil de Notingan que mide aspectos multidimensionales, es empleado en personas de edad. El factor económico es importante en la calidad de vida, en todos sus ámbitos. Es un ingrediente de la calidad de vida, no sólo en la vejez, en todas las edades. Las medidas de calidad de vida suelen medir este factor. Si se encuentran bien, con autonomía, queda más estimado el factor económico. Es una dimensión de la calidad de vida. En la calidad de vida, el nivel económico, suele medirse de forma subjetiva. La independencia implica autonomía.

La satisfacción vital es una de las dimensiones últimas de la calidad de vida. Es imposible que una persona se siente bien en salud y autonomía y esté aislada. El amor es importante aquellas personas que tiene mayor calidad de vida tienen más satisfacción vital, buena salud y autonomía, suelen darse todos los factores conjuntamente.

La pérdida de la pareja, de un ser querido aumenta la mortalidad y el suicidio. Disminuye la salud, la red de apoyo disminuye notablemente sobre todo en la vejez, la pareja es el lazo más fuerte de apoyo socio-familiar en la vejez, los parientes, amigos se van perdiendo, e incluso los hijos no en términos de mortalidad pero sí de distancia.

SEGUNDA ENTREVISTA. «La calidad de vida» es vivir bien, no tener ningún tipo de problema ni físico ni social ni psicológico. Salud dinero y amor en todas las etapas de la vida. En la vejez es más salud y autonomía con nitidez y rotundidad. La economía es determinante para la salud, lo condiciona todo, es una variable estructural de la salud. Los recursos económicos, en la vejez, son condicionantes para la salud. Las pensiones son bajas y las personas se adaptan bien, pocas vividas con pocos recursos ayudan y hacen aprender y encontrar los pocos recursos como suficientes para adaptarse y organizar su vida con satisfacción. Los viejos tiene coberturas sociales que antes no se pensaban, por tanto, sus expectativas son mejores, seguridad en la prestaciones, atención a los problemas de salud. Es habitual que viajen, que salgan, se ha potenciado el sociacionismo. Esto recursos no lo tuvieron sus padres.

La perdida de la pareja es un predictor importante en la calidad de vida. Influye en el modo de vida de forma negativa. Afecta negativamente a la salud.

Las mujeres tiene un comportamiento distinto en la sociedad, las mujeres cuidan la salud y buscan la soluciones de salud de los hijos y de la pareja. Además hay más redes de apoyo en las viudas que en los viudos. Las mujeres tienen más recursos para autocuidarse por su rol social. El entrenamiento en «saberte cuidar» ayuda a saber vivir en la vejez.

TERCERA ENTREVISTA. «Calidad de vida» es personal, individual, es difícil medir, necesidades cubiertas físicas, psíquicas y sociales. En la vejez hay unos de- terioros físicos que puede conducir si no tiene unos apoyos familiares y sociales ha tener una disminución notable de salud. El apoyo social es importante para mantener una calidad de vida en la vejez. La calidad de vida en la vejez es tener salud y la salud es independencia, funcionalidad y además redes de apoyo familiar y social. Es importante para la calidad de vida en la vejez de una preparación previa, de un aprendizaje pues ayuda a ser feliz a tener tu vida preparada para el ocio y otras actividades que te satisfagan. La pérdida de la pareja es importante y sobre todo si la vida de pareja ha sido de apoyo, de amistad. Ayuda a ser feliz. El sexo determina básicamente el aprendizaje, la independencia, el hombre por su papel social pierde no sólo su pareja si no la persona que le ha facilitado su vida.

El factor económico es importante en cualquier edad, si no se cubren las necesidades se dificulta la vida. Para tener calidad de vida tiene que tener cubiertas todas sus necesidades.

Los factores más importante que determinan la calidad de vida en la vejez, por orden de importancia, son: la salud, la autonomía, el refuerzo socio-familiar y el factor económico.

CUARTA ENTREVISTA. «La calidad de vida» es un concepto amplio, su base es tener cubiertas las necesidades básicas pero teniendo sentido. Comer no es igual a tragar, el organismo se resiente, igual cuando respiramos acto continuo de relajación, el sueño puede o no ser reparador. Necesidades básicas cubiertas de forma satisfactoria. Aceptando nuestro cuerpo, trabajando la relación con nuestro cuerpo sin mensajes de atención al cuerpo, sin comunicación alguna no es la vía que conduce a la satisfacción. Aceptando que nuestro cuerpo va cada día envejeciendo, si no se acepta, vivimos en una continua ansiedad, perdiendo atractivos que la gente valora.

Aspecto importante en la calidad de vida son:

- «Cómo se acepta la vida». No son las cosas lo que nos hacen daño sino la manera de verlas problemas. Buscar la forma de disminuir la ansiedad y los recursos como la relajación para que la vida nos la tomemos mejor.

- Tener objetivos, metas, que nos ayude a vivir a ilusionarnos. Cuando no hay objetivos ¿qué queda?, la persona se deprime. Es importante mantener las metas.

- Favorer la autoestima, es decir, que la persona se quiera, es muy valioso incluso con sufrimiento. El sufrimiento como parte de la vida aceptándolo como un valor, no como una carga puede favorecer la calidad de vida.

- También la ética, la moral, los valores, si los tenemos y practicamos nos da sentido a la vida. Nos ayuda a reestructurarnos la vida. El amor, la honestidad, la sinceridad... Los valores hacen que la persona se resigne para vivir la vida.

- La relación social con personas animales plantas y las cosas. Si la persona se aísla termina hundiéndose, deprimiéndose. 
- Aceptar los roles sociales, con su edad, pretendiendo sólo aquello que para su edad la sociedad espera de él.

- Mantener los compromisos sociales de pareja, de madre sin imponer la forma de vida a los demás, aceptando el nuevo rol. Ser consecuente con su papel social.

- Disfrutar del tiempo libre, organizándose el tiempo para sentirse a gusto, bien.

- Debemos aceptar la enfermedad «no para resignarse», sino, para luchar para recobrar la salud. La persona debe ser agente de su propia salud, de su vida. La enfermedad debe vivirla bien.

\section{CONCLUSIONES}

1) La calidad de vida es difícil de cuantificar porque involucra dimensiones personales subjetivas muy difíciles de definir. Podemos mediante la creación de indicadores explicar las diferencias en la satisfacción con datos objetivos, pues la satisfacción puede crecer cuanto más binestar se posea. Los indicadores son unidades observables de un fenómeno social que establece el valor de un rango que no se puede observar ni cuantificar directamente. Los elementos o factores que podemos cuantificar son: recursos economicos, salud física, incapacidades funcionales, recursos familiares y sociales, vivienda, actividad y ocio.

2) Podríamos resaltar que la calidad de vida en la vejez está muy relacionada con el grado de funcionalidad, es decir, de independencia física, psíquica y social de la persona. Cuando dependemos de otra persona, aunque sea sumamente querida, nos sentimos en desventaja, relegados.

Si la dependencia es física, por mucho amor que se nos dispense, siempre te consideras una carga a no ser que el sufrimiento se acepte como una valor moral ético que de sentido a la vida. Cuando preguntas miedos o temores en la pérdida de salud. La mayoría de las personas te contestan que lo que más temen es depender y ser carga para su familia y/o sociedad. El sufrimiento, generalmente, no es aceptado, te resignas y buscas alternativas para darle sentido, pero creemos que no es compatible con la felicidad. Si la dependencia es psíquica la persona no tiene muchas veces la capacidad de juicio como para considerarse una carga y si quien la atiende es amable y respetuosa la persona dependiente puede sentirse feliz.

La persona con dependencia socioeconómica no se puede sentir bien, todos deseamos una cuantía que nos permita vivir con libertad y nos permita un bienestar, la dependencia limita la libertad y aumenta la gratitud y la complacencia de la persona a quien o quienes son sus benefactores y, aunque, se sienta feliz, seguro que añoraria ser el benefactor y no el menesteroso.

3) Un factor que influye en la disminución de calidad de vida es: la muerte de un miembro de la pareja sobre todo en el caso del fallecimiento de la mujer. El rol social tradicional de la mujer ha consistido en el cuidado de la salud, alimentación, higiene, etc. Al fallecer la es- posa, el marido suele descuidar estos aspectos que son determinantes en su salud, además de sentirse dependiente de los hijos y/o de otras personas, pues, no está entrenado en las tareas de la casa.

4) Consideramos el estado de salud percibida el factor más importante de la calidad de vida en la vejez. Las personas consideran el estado de salud como uno de los bienes más preciados e importantes en su vida, sobre todo, cuando esta disminuye o se pierde. Siempre añoran, ¿si estuviera mejor?, ¿si pudiera?, ¿si me llevaran las piernas?, ¿si no me fatigase? Todo son lamentaciones y deseos de recuperación, por tanto, aunque se sea feliz, siempre aumentaría esa felicidad si se consiguiera una restitución de su estado de salud.

5) En cuanto a la economía podríamos resaltar que las personas mayores se sienten felices con recursos muy escasos, pero suficientes para sus aspiraciones, esto podría explicarse por:

a) Etapas anteriores vividas en condiciones de extrema escasez y por la ausencia de pensiones en la época de sus antecesores.

c) Escala de valores, menor, que la población más joven sobre las diferentes necesidades y en especial de las necesidades de ocio y consumo.

d) La seguridad en las prestaciones les garantiza la satisfacción de sus necesidades básicas de alimenta-ción, vestido y vivienda de por vida.

\section{BIBLIOGRAFÍA}

Confort, A. (1986) Una buena edad la tercera edad. Blume, Barcelona.

Langarica, R. (1985) Geriatría y gerontología. Interamericana, MJxico.

Reig, A., Ribera, D. (1992) Perspectivas en Gerontología y Salud. Promolibro, Valencia.

Ribera, D.; Majos, A.; Reig, A. (1993) La Cuarta Edad Europea: Envejecer en la Costa Blanca. Fundación Caja Madrid, Barcelona.

Richart, M. (1995) Determinantes biopsicosociales de la calidad de vida en una muestra comunitaria de ancianos: Un estudio longitudinal de tres años. Tesis doctoral. Facultad de PsicologRa, Universidad de Murcia.

Skiner, B.J. \& Vaugha, M. E. (1986). Disfrutar la vejez. Martinez roca, Barcelona.

Buendia,J. y Riquelme, A. (1993). Implicaciones de las condiciones socioeconómicas y del apoyo social en la salud mental de la población. Psiquis 14,35-48

Freixas,A. (1992). Mujer y envejecimiento. Aspectos psicológicos. Revista del colegio oficial de psicólogos de Andalucía Occidental 36, 39-45

Coriel,J. \& Levin, J. \$ Gartl y Jaco, E. (1992). Estilo de vida. Un concepto emergente en las ciencias sociomédicas. Clínica y salud 1, 221-231. 